Research Paper

\title{
Gene Expression Profiling Reveals Novel Candidate Markers of Ovarian Carcinoma Intraperitoneal Metastasis
}

\author{
Katerina Elsnerova1, 2,3凶 , Alena Bartakova4, Josef Tihlarik ${ }^{4}$, Jiri Bouda ${ }^{4}$, Lukas Rob ${ }^{5}$, Petr Skapa ${ }^{6}$, Martin \\ Hruda $^{5}$, Ivan Gut ${ }^{1}$, Beatrice Mohelnikova-Duchonova1, 7, Pavel Soucek1,3, Radka Vaclavikova1,3 \\ 1. Toxicogenomics Unit, National Institute of Public Health, Srobarova 48, 10042 Prague 10, Czech Republic; \\ 2. Third Faculty of Medicine, Charles University, Ruska 87, 10000 Prague 10, Czech Republic; \\ 3. Biomedical Center, Faculty of Medicine in Pilsen, Charles University, Alej Svobody 76, 32300 Pilsen, Czech Republic; \\ 4. Department of Gynecology and Obstetrics, Faculty of Medicine and University Hospital in Pilsen, Charles University, Alej Svobody 80, 304 60 Pilsen, Czech \\ Republic; \\ 5. Department of Gynecology and Obstetrics, Third Faculty of Medicine and Vinohrady University Hospital, Charles University, Srobarova 50, 10034 Prague \\ 10, Czech Republic; \\ 6. Department of Pathology and Molecular Medicine, Second Faculty of Medicine and Motol University Hospital, Charles University, V Uvalu 84,15006 \\ Prague 5, Czech Republic; \\ 7. Department of Oncology, Palacky University Medical School and University Hospital, I. P. Pavlova 6, 77900 Olomouc, Czech Republic.
}

$\square$ Corresponding author: Katerina Elsnerova, Toxicogenomics Unit, National Institute of Public Health, Srobarova 48, 10042 Prague 10, Czech Republic, phone: +420 267082633; fax: +420 267311236; e-mail: katerina.elsnerova@szu.cz

( ) Ivyspring International Publisher. This is an open access article distributed under the terms of the Creative Commons Attribution (CC BY-NC) license (https://creativecommons.org/licenses/by-nc/4.0/). See http://ivyspring.com/terms for full terms and conditions.

Received: 2017.04.27; Accepted: 2017.09.11; Published: 2017.10.09

\begin{abstract}
Epithelial ovarian cancer (EOC) has the highest mortality among gynecological carcinomas. The lack of specific markers for prognostic determination of EOC progression hinders the search for novel effective therapies.

The aim of the present study was (i) to explore differences in expressions of ATP-binding cassette $(A B C)$ and solute carrier (SLC) transporter genes, genes associated with drug metabolism and cell cycle regulation between control ovarian tissues $(n=14)$, primary EOCs $(n=44)$ and intraperitoneal metastases $(n=29)$; (ii) to investigate associations of gene expression levels with prognosis of patients with intraperitoneal metastases.

In all tissue samples, transcript levels of the above target genes were assessed using quantitative real-time PCR. Gene expression levels were compared between particular tissue types and evaluated with regard to progression-free survival (PFS) and drug-resistance status of patients with metastases.

Gene expression of $A B C A 7$ significantly increased and that of ESR2 decreased in the order control ovarian tissues - primary EOCs - metastases. High expressions of $A B C A 2 / 8 / 9 / 10, A B C B 1, A B C C 9$, $A B C G 2, A T P 7 A, S L C l 6 A 14$, and SOD3 genes were significantly associated with longer progression-free survival of patients. In intraperitoneal metastases, expression of all of these genes highly correlated and indicated prognostic profile. Transporters from the ABCA family, ABCG2, and ESR2 are involved mainly in lipid metabolism, membrane transport, and cell proliferation. These processes are thus probably the most important for EOC progression.

Based on these results, we have proposed novel markers of ovarian carcinoma progression and metastatic spread which might be potentially useful as therapeutic targets. Their significance should be further explored on a larger independent set of patients.
\end{abstract}

Key words: epithelial ovarian cancer, progression, metastases, markers, gene expression. 


\section{Introduction}

Epithelial ovarian cancer (EOC) is the most lethal gynecological carcinoma. EOC is often diagnosed at advanced stages (i.e. stage III and IV according to International Federation of Gynecology and Obstetrics, FIGO) when patients have already presented with intraperitoneal metastases and have an extremely high risk of developing distant metastases. Since development of metastases is the leading cause of cancer-related deaths worldwide [1], five-year overall survival (OS) of ovarian cancer patients is $<50 \%$ [2]. Deciphering key genetic factors contributing to metastatic spread of EOC would greatly improve prognosis and provide targets for therapeutic design.

Ovarian tumor cells can spread via intraperitoneal dissemination or via expansion of tumor cells into blood or lymphatic circulation [3]. The first one happens through passive circulation of ascitic fluid to other organs in peritoneal cavity. This way used to be considered the most frequent one; however, recent findings have shown that hematogenous dissemination is also a very important way for EOC metastatic spread. Unlike the passive dissemination, the process of hematogenous spread makes the tumor cells different from primary EOC cells [4]; they differ mostly in terms of markers of epithelial-mesenchymal transition (EMT) [3] which is the key step in EOC metastasis [4]. However, they might also differ with respect to other characteristics suggesting differences in character and behavior depending on the stage of tumor progression.

An example of an EMT marker in ovarian cancer cells is epidermal growth factor receptor (EGFR) [5]. Hyperactive EGFR signaling was shown to associate with increased invasiveness in ovarian cancer cells that were resistant to cisplatin [6]. Another receptor, the avian erythroblastic leukemia viral oncogene homolog 2 (ERBB2) protein is increased in advanced stage tumors; moreover, association of this protein with survival of EOC patients has been proposed [7]. A potential role for oxidative stress response in behavior of ovarian tumors was previously suggested by the observed deregulation of superoxide dismutase 2 (SOD2) and glutaredoxin (GLRX) genes in ovarian cancer patients with unfavorable outcomes [8].

An increased expression of p53 and Ki67 proteins (encoded by TP53 and MKI67 genes, respectively) was found in more aggressive ovarian cancer types and in advanced stage tumors [9]. This finding supports an earlier work in which the presence of either p53 protein or p53-antibody in ascites from ovarian cancer patients was demonstrated [10]. Since ascitic fluid has an important role in EOC metastasis [4], p53 could be involved in that process.

ATP-binding cassette $(\mathrm{ABC})$ and solute carrier (SLC) transporters have recently been linked to drug resistance [11] and ovarian cancer outcome [12, 13]. Similarly, copper-transporting ATPases ATP7A and $A T P 7 B$ have been shown to modulate drug resistance in ovarian cancer $[14,15]$ and silencing of $A T P 11 B$ resulted in reduced growth of ovarian tumor in vivo [16]. However, little is known about the role of $A B C$, SLC, and other transporters in EOC progression and metastases development.

Nuclear receptors, e.g., estrogen receptor beta (ER $\beta$, ESR2), small heterodimer partner (SHP, NR0B2), farnesoid X-activated receptor (FXR, NR1H4) or vitamin D receptor (VDR, NR1I1) are involved in regulation of several cellular processes such as proliferation or apoptosis [17, 18]. However, information on the function of nuclear receptors in EOC progression is limited.

In this study, we evaluated differences in gene expression of 62 membrane transporters, genes associated with drug metabolism, and cell cycle regulating genes among samples of control ovarian tissues, primary EOCs, and intraperitoneal metastases. We focused on significant tendencies in gene expression levels in particular tissue types, and on associations of gene expression with drug-resistance status and progression-free survival (PFS) in patients with intraperitoneal metastases. We aimed to provide a hypothesis generating screen for further studies on the importance of genetic markers of EOC metastasis that would be potentially useful as prognostic markers and novel drug targets.

\section{Patients and Methods}

\section{Patients}

Study comprised of tissue samples from 63 patients with ovarian carcinoma. Of these, paired samples of primary tumor and EOC intraperitoneal metastases were available for 10 patients. Other 34 patients provided primary ovarian carcinomas, and EOC intraperitoneal metastases were collected from other 19 patients. Patients were diagnosed in the University Hospital in Pilsen, Czech Republic during 2013 - 2016. As a control set, 14 samples of ovarian tissues without presence of tumor cells from Motol University Hospital in Prague, Czech Republic were used. The patients who provided control ovarian 
tissues attended hospital for various gynecological disorders excluding EOC. The tissue samples were fresh-frozen and stored at $-80^{\circ} \mathrm{C}$ until processing. All samples were histopathologically examined according to standard diagnostic procedures.

All patients provided an Informed Consent in accordance with the requirements of the Ethical Commission of the National Institute of Public Health in Prague, University Hospital in Pilsen and Motol University Hospital in Prague, Czech Republic. The study was in accordance with the Declaration of Helsinki.

\section{Isolation of total RNA and preparation of cDNA}

Fresh-frozen tissue samples were grounded to powder by mortar and pestle under liquid nitrogen. RNA was isolated using AllPrep DNA/RNA/Protein Mini Kit (Qiagen, Hildesheim, Germany) according to manufacturer's protocol. Total RNA was quantified by the help of Quant-iT RiboGreen RNA Assay Kit (Invitrogen, Eugene, OR) and cDNA was synthesized using RevertAid First Strand cDNA Synthesis Kit (MBI Fermentas, Vilnius, Lithuania). Quality of cDNA was checked by PCR as described previously [19].

Subsequently, cDNA was preamplified. Preamplification mixture contained 5x PerfeCTa PreAmp SuperMix (Quanta Biosciences, Gaithersburg, MD), pool of TaqMan Gene Expression Assays (0.2x each; Life Technologies, Foster City, CA, listed in Table S1), 10-times diluted cDNA and nuclease-free water in total volume of $25 \mu \mathrm{l}$. Fourteen preamplification cycles were used according to the manufacturer's protocol. The preamplified cDNA was stored at $-20^{\circ} \mathrm{C}$ until real-time PCR was performed.

\section{Quantitative real-time PCR}

To determine relative gene expression, ViiA7 Real-Time PCR System (Life Technologies) was used. The real-time PCR study design adhered to the Minimum Information for Publication of Quantitative Real-Time PCR Experiments Guidelines [20]. Reaction mixture contained 2x TaqMan Gene Expression Master Mix (Life Technologies), 20x TaqMan Gene Expression Assay specific for each gene of interest (Life Technologies; listed in Table S1), $2 \mu 1$ of cDNA and nuclease-free water in total volume of $5 \mu \mathrm{l}$. Sample cDNA was diluted 32-times in TE buffer. For each assay, negative control with nuclease-free water instead of cDNA was used.

To achieve the best reaction efficiency (> $90 \%$ ), cycling conditions of each assay were optimized using a calibration curve as described previously [12].

Cycling conditions were initial hold at $50^{\circ} \mathrm{C}$ for 2 min, hold at $95{ }^{\circ} \mathrm{C}$ for $10 \mathrm{~min}$ and then 45 cycles consisting of denaturation at $95{ }^{\circ} \mathrm{C}$ for $15 \mathrm{sec}$ and annealing/extension at $60{ }^{\circ} \mathrm{C}$ for $60 \mathrm{sec}$ (see Table S1 for exceptions). Samples were analyzed in duplicates; those with standard deviation larger than $0.5 \mathrm{Ct}$ were reanalyzed.

PPIA, UBC and YWHAZ genes were used as reference genes for normalization of results based on their stability in ovarian tissue assessed previously by NormFinder and geNorm software [12]. All gene expression data were submitted to the Gene Expression Omnibus public data repository (http://www.ncbi.nlm.nih.gov/geo) under the accession number GSE102180.

\section{Data analysis}

All statistical analyses were performed in SPSS v16.0 program (SPSS Inc, Chicago, IL). First, differences in relative expression level between control tissues, primary tumors and metastases were evaluated by the Kruskal-Wallis non-parametric test. Then, differences between primary ovarian carcinomas and metastases were evaluated by the Mann-Whitney non-parametric test. The false discovery rate (FDR) test was applied according to Benjamini and Hochberg [21] using FDR online calculator [22] and adjusted p-values were computed for each comparison.

Next, associations of gene expression levels in metastases with patients' clinical data were studied. The Kruskal-Wallis test was used to evaluate drug resistance phenotype in patients who presented progression, recurrence or death in less than six months (resistant), in more than six months, but less than 12 months (intermediate), or in more than 12 months (sensitive) since the termination of first line chemotherapy treatment [23]. Also patients who did not present progression, recurrence or death for at least 12 months were considered chemotherapy-sensitive.

The log-rank test and Kaplan-Meier plot were employed to identify significant associations of gene expression level with PFS. Then hazard risk (HR) of relapse with 95\% confidence interval (CI) was computed by the Cox regression. Relative expressions of genes which showed significant result in the log-rank test were correlated using the Spearman test. PFS was estimated as time from surgical tumor removal to progression, recurrence, death, or to the last examination without evidence of any disease signs.

A two-sided p-value of less than 0.05 was considered statistically significant for all statistical analyses. 


\section{Results}

\section{Patients' characteristics}

Clinical data from patients who provided tissue samples of either primary ovarian tumor $(n=44)$ or intraperitoneal metastases $(n=29)$ are shown in Table 1 . For the purpose of comparing gene expression level, 14 control ovarian tissue samples were used. Control ovarian samples were reviewed by a pathologist, and samples without presence of tumor cells were further processed.

The median age ( \pm standard deviation, $S D)$ at the time of diagnosis of patients with primary EOC, patients with intraperitoneal metastases and patients who provided control samples, was $62.5 \pm 9.9,62.0 \pm$ 12.7 , and $53.5 \pm 13.3$ years, respectively. The median PFS $( \pm \mathrm{SD})$ of patients with primary EOCs and metastases was $15.0 \pm 10.3$ and $12.0 \pm 8.9$ months, respectively.

Table 1. Clinical characteristics of patients with primary ovarian tumors or intraperitoneal EOC metastases

\begin{tabular}{|c|c|c|c|}
\hline \multirow[t]{2}{*}{ Characteristics } & Primary EOC & EOC metastases & \multirow[t]{2}{*}{ p-Valuec } \\
\hline & $\mathrm{N}(\%)^{\mathrm{a}}$ & $\mathrm{N}(\%)^{\mathrm{a}}$ & \\
\hline \multicolumn{4}{|l|}{ FIGO stage } \\
\hline $\mathrm{I}+\mathrm{II}$ & $13(30.2)$ & 0 & \multirow[t]{3}{*}{0.001} \\
\hline $\mathrm{III}+\mathrm{IV}$ & $30(69.8)$ & $28(100.0)$ & \\
\hline data not available & 1 & 1 & \\
\hline \multicolumn{4}{|l|}{ Histological grade } \\
\hline 1 & $6(14.3)$ & 0 & \multirow[t]{3}{*}{0.076} \\
\hline $2+3$ & $36(85.7)$ & $26(100.0)$ & \\
\hline data not available & 2 & 3 & \\
\hline \multicolumn{4}{|l|}{ EOC type } \\
\hline HGSC $^{b}$ & $30(68.2)$ & $22(84.6)$ & \multirow[t]{3}{*}{0.163} \\
\hline other types & $14(31.8)$ & $4(15.4)$ & \\
\hline data not available & 0 & 3 & \\
\hline \multicolumn{4}{|c|}{ First-line chemotherapy } \\
\hline administered & $39(88.6)$ & $27(93.1)$ & \multirow[t]{2}{*}{0.696} \\
\hline not administered & $5(11.4)$ & $2(6.9)$ & \\
\hline \multicolumn{4}{|c|}{ Progression, recurrence or death } \\
\hline yes & $19(43.2)$ & $18(62.1)$ & \multirow[t]{2}{*}{0.153} \\
\hline no & $25(56.8)$ & $11(37.9)$ & \\
\hline \multicolumn{4}{|l|}{ Drug resistance status } \\
\hline resistant & $5(19.2)$ & $7(38.9)$ & \multirow[t]{4}{*}{0.170} \\
\hline intermediate & $5(19.2)$ & $5(27.8)$ & \\
\hline sensitive & $16(61.5)$ & $6(33.3)$ & \\
\hline data not available & 18 & 11 & \\
\hline
\end{tabular}

aNumber of patients with percentage in parentheses

bHGSC $=$ high grade serous carcinoma

'Significance of difference between both sets of patients by the Fisher's Exact test

\section{Comparison of gene expression in controls, primary carcinomas and metastases}

The present study compared expression of 62 genes in control ovarian tissues, samples of primary ovarian carcinomas, and samples of EOC metastases. Ten genes ( $A B C A 10, A B C D 3, A B C E 1, A B C F 3, A B C G 2$, ESR2, NR1H4, SLC16A14, SLC22A5, and TRAP1) showed a significantly decreasing tendency in expression levels from controls to primary tumors to metastases $(\mathrm{C}>\mathrm{pT}>\mathrm{M})$. Ten genes $(A B C A 7 / 12 / 13$, $A B C B 2 / 3 / 11, \quad A B C C 3, \quad N R 111, \quad S L C 22 A 18$, and SLC31A1; Table S2A) showed an opposite tendency in gene expression levels $(\mathrm{C}<\mathrm{pT}<\mathrm{M})$. Except for $A B C A 12$ gene, all observed deregulations passed the FDR correction for multiple testing (Table 2A).

\section{Comparison of gene expression in primary carcinomas and metastases}

Differences in relative transcript levels of the genes of interest in primary ovarian carcinomas and metastases were also evaluated. Two genes (ESR2, NR1H4) were down-regulated and 12 genes $(A B C A 1 / 7, A B C B 2, A B C C 2 / 3 / 9, A B C D 1 / 2, G L R X$, NR1I1, SLC31A2, and SOD2; Table S2B) were up-regulated in metastases when compared with primary carcinomas. Among these, down-regulation of ESR2 and up-regulation of $A B C A 7, A B C C 2, G L R X$, and SLC31A2 passed the FDR correction for multiple testing (Table 2B).

Due to the small sample number it was not possible to perform sensible comparison of gene expression level in either paired or unpaired samples of primary tumors and metastases. However, we provide the results of this comparison in the Table S3.

Table 2. Significant differences in gene expression levels between (A) controls, primary EOC tumors and intraperitoneal metastases; (B) primary tumors and metastases

\begin{tabular}{|c|c|c|c|c|}
\hline & (A) & & (B) & \\
\hline Gene & p-Value ADJ & Trend & $p$-Value ADJ & Trend \\
\hline$A B C A 7$ & $<0.001$ & $\mathrm{C}<\mathrm{pT}<\mathrm{M}$ & 0.018 & pT $<$ M \\
\hline ABCA10 & $<0.001$ & $\mathrm{C}>\mathrm{pT}>\mathrm{M}$ & NS & \\
\hline ABCA13 & 0.001 & $\mathrm{C}<\mathrm{pT}<\mathrm{M}$ & NS & \\
\hline$A B C B 2$ & 0.004 & $\mathrm{C}<\mathrm{pT}<\mathrm{M}$ & NS & \\
\hline$A B C B 3$ & $<0.001$ & $\mathrm{C}<\mathrm{pT}<\mathrm{M}$ & NS & \\
\hline ABCB11 & 0.043 & $\mathrm{C}<\mathrm{pT}<\mathrm{M}$ & NS & \\
\hline$A B C C 2$ & NS & & 0.018 & pT $<$ M \\
\hline$A B C C 3$ & $<0.001$ & $\mathrm{C}<\mathrm{pT}<\mathrm{M}$ & NS & \\
\hline$A B C D 3$ & $<0.001$ & $\mathrm{C}>\mathrm{pT}>\mathrm{M}$ & NS & \\
\hline$A B C E 1$ & $<0.001$ & $C>p T>M$ & NS & \\
\hline$A B C F 3$ & $<0.001$ & $C>p T>M$ & NS & \\
\hline$A B C G 2$ & $<0.001$ & $\mathrm{C}>\mathrm{pT}>\mathrm{M}$ & NS & \\
\hline ESR2 & $<0.001$ & $\mathrm{C}>\mathrm{pT}>\mathrm{M}$ & 0.018 & $\mathrm{pT}>\mathrm{M}$ \\
\hline GLRX & NS & & 0.018 & $\mathrm{pT}<\mathrm{M}$ \\
\hline NR1H4 & $<0.001$ & $\mathrm{C}>\mathrm{pT}>\mathrm{M}$ & NS & \\
\hline NR111 & $<0.001$ & $\mathrm{C}<\mathrm{pT}<\mathrm{M}$ & NS & \\
\hline SLC16A14 & $<0.001$ & $\mathrm{C}>\mathrm{pT}>\mathrm{M}$ & NS & \\
\hline SLC22A5 & 0.004 & $\mathrm{C}>\mathrm{pT}>\mathrm{M}$ & NS & \\
\hline SLC22A18 & 0.025 & $\mathrm{C}<\mathrm{pT}<\mathrm{M}$ & NS & \\
\hline SLC31A1 & 0.020 & $\mathrm{C}<\mathrm{pT}<\mathrm{M}$ & NS & \\
\hline SLC31A2 & NS & & 0.009 & $\mathrm{pT}<\mathrm{M}$ \\
\hline TRAP1 & $<0.001$ & $\mathrm{C}>\mathrm{pT}>\mathrm{M}$ & NS & \\
\hline
\end{tabular}

p-Value ADJ $_{\text {by }}$ the Kruskal-Wallis (comparison C vs. pT vs. M) or the Mann-Whitney (comparison $\mathrm{pT} v s . \mathrm{M}$ ) tests adjusted by the FDR test

$\mathrm{C}=$ controls, $\mathrm{pT}=$ primary tumors, $\mathrm{M}=$ metastases

NS $=$ not significant

All genes have been analyzed in both comparisons (Table S2), but to retain concise style only significant values passing the FDR adjustment are displayed here. 


\section{Associations of gene expression level in metastases with drug resistance and PFS}

Due to the fact that clinical data from patients with intraperitoneal metastases had uniform character (stage III/IV, grade 3, high grade serous carcinoma; see Table 1), only the associations with drug resistance and PFS were tested in the set.

None of the genes of interest showed any relationship with patient drug resistance status. On the other hand, expressions of ten genes (ABCA2/8/9/10, ABCB1, ABCC9, ABCG2, ATP7A, $S L C 16 A 14$, and SOD3) were significantly lower in intraperitoneal metastases of patients with worse PFS. Except for $A B C B 1$ and $A B C G 2$, these associations remained significant after the Cox regression was computed $(\mathrm{p}<0.05, \mathrm{HR}>4.7, \mathrm{CI}=1.23$ - 19.19; Table S4; examples of Kaplan-Meier plots are shown in Figure 1).

In order to explore potential mutual deregulation of the followed genes, additional analyses have been performed separately in metastases, primary tumors, and control tissues. These analyses have revealed that expression levels of the above described genes mutually correlated in intraperitoneal metastases ( $p<0.05$ for all genes). The same trend was observed in primary tumors, but not in control ovarian samples (Figure 2).

\section{Discussion}

Advanced EOC presents a challenge due to the absence of predictive markers of disease risk useful for early diagnosis and lack of prognostic and predictive factors for therapy individualization. As a result, late diagnosis, metastatic spread, and frequent occurrence of resistance to therapy lead to a five-year relative survival rate of $44 \%$ [2]. This study searched for candidate genes with putative prognostic value in EOC progression that were selected from functionally-relevant groups of cancer-related processes.

Twenty genes were significantly deregulated in the sequence of control samples, primary tumors, and EOC metastases $(C-p T-M)$. Nineteen of these deregulated genes remained significant after the FDR correction for multiple testing. Except for $A B C B 11$ and SLC31A1, the other genes also showed deregulation in primary EOCs when compared with controls in our previous study on an independent sample set [12]. In comparison of relative expression level in primary carcinomas and metastases, 14 genes were differently expressed in the present study. Five of them remained significant after the FDR correction. The deregulation in metastases compared to primary tumors, but not in comparison of $\mathrm{C}-\mathrm{pT}-\mathrm{M}$, suggests a potential role of these genes in metastatic spread of EOC.

Taken together, six genes were consistently deregulated both in the sequence of $\mathrm{C}-\mathrm{pT}-\mathrm{M}$ and in the comparison of primary carcinomas compared with metastases only (Figure 3). For $A B C A 7$ and ESR2 genes, these deregulations were significant after the FDR correction. These results seem to represent true associations, and the genes might play an important role in EOC progression.
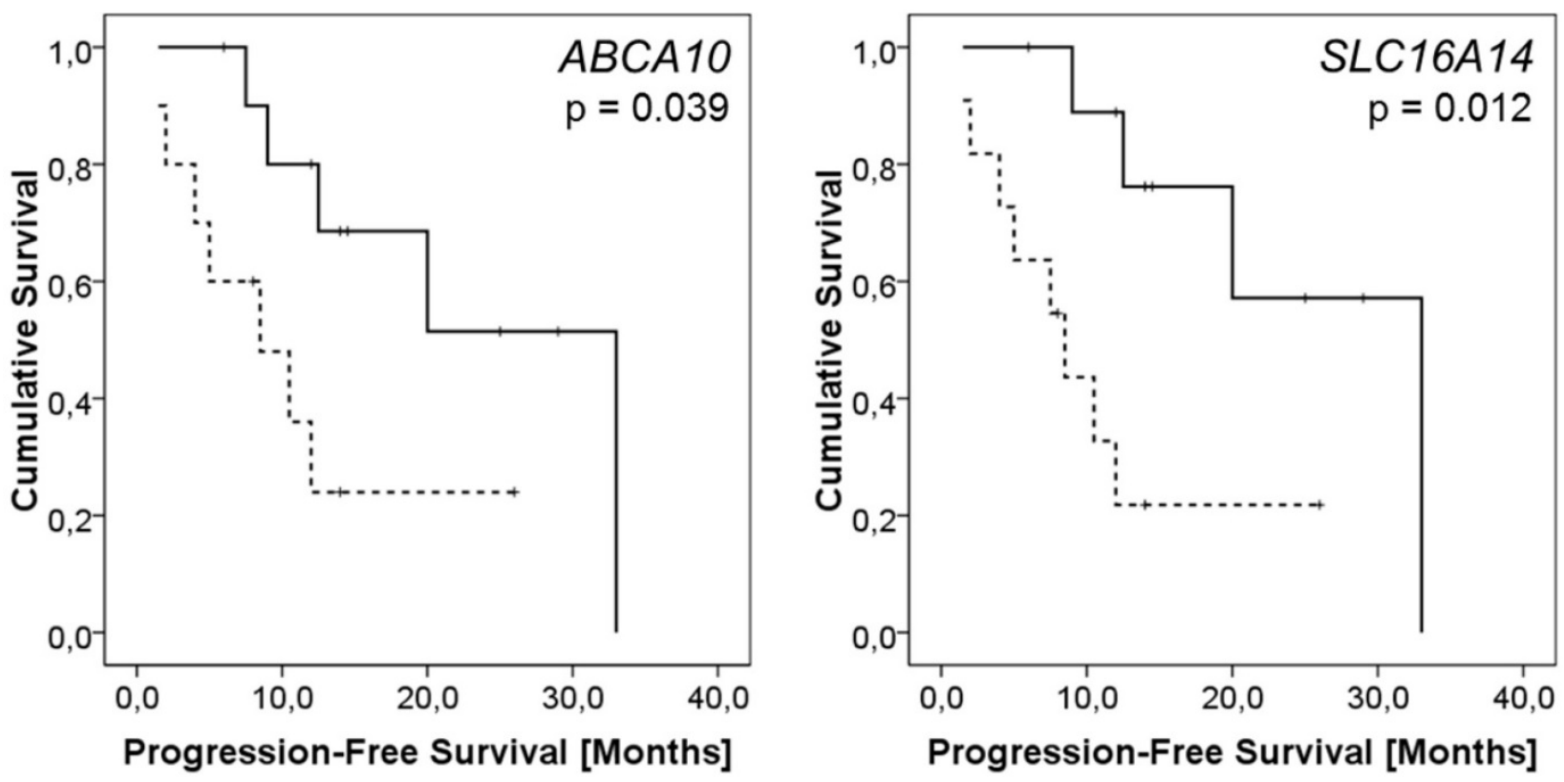

Figure 1. Kaplan-Meier plots representing results of survival analysis of ABCAIO and SLCI6AI4 genes. Survival curves for patients with the intratumoral ABCA I 0 or SLCI 6AI 4 expression levels above the median (solid line) vs. patients with lower expression than the median (dashed line) are displayed. The difference in the mean PFS between the compared groups of patients was evaluated by the log rank test. 

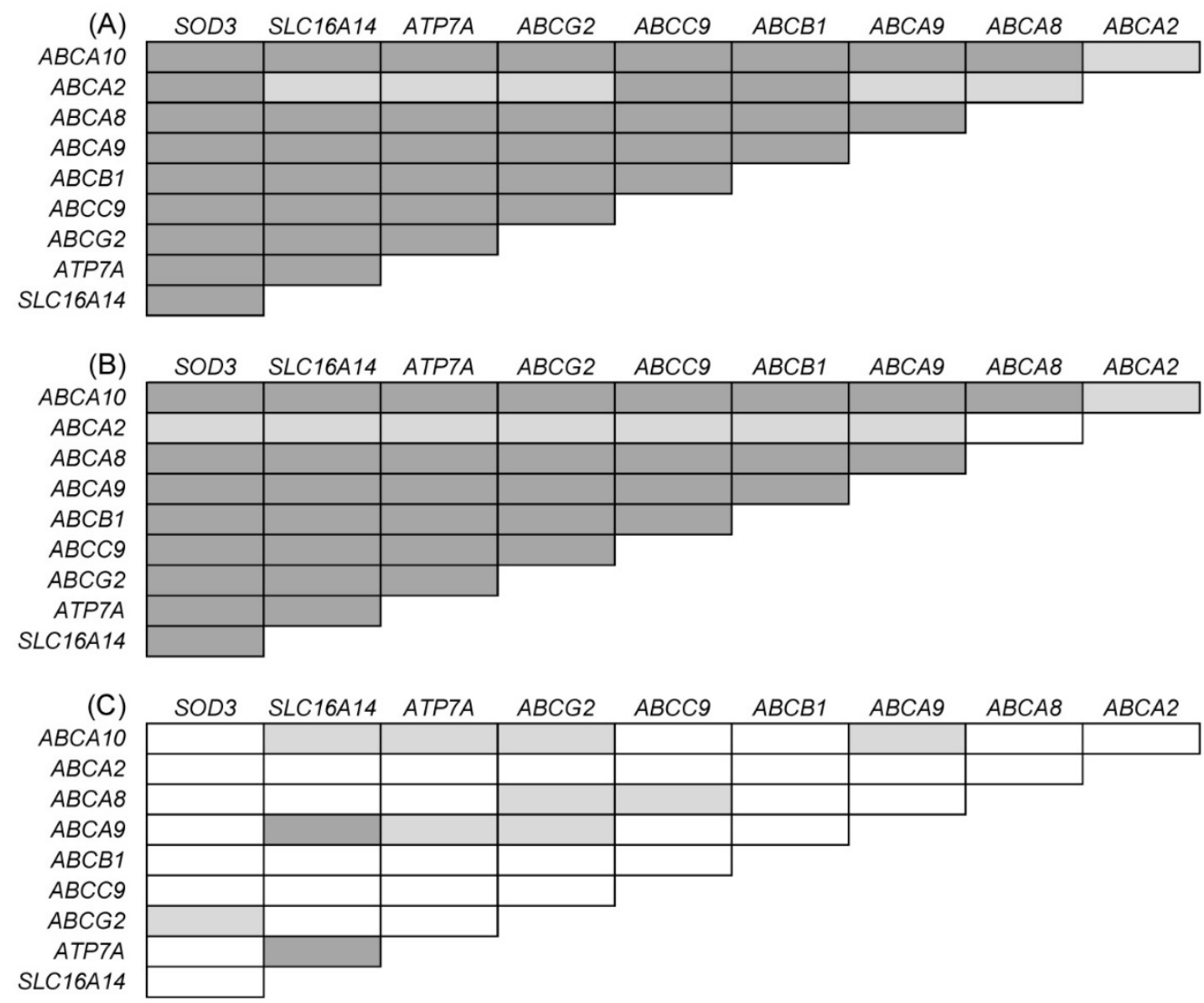

Figure 2. Mutual correlations of gene expression levels in (A) intraperitoneal EOC metastases; (B) primary tumors; (C) control tissues. Only genes that were significantly associated with PFS of patients with EOC metastases were evaluated. Associations highlighted in grey are significant. Dark grey: $\mathrm{p}<0.001$; light grey: $p<0.005$

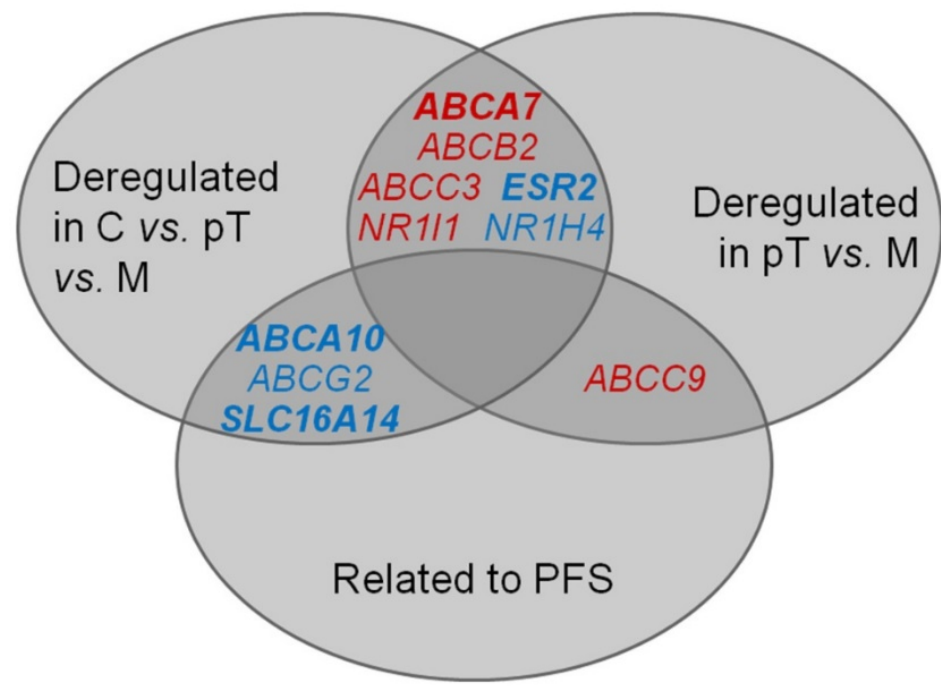

Figure 3. Intersection of significant results of (i) comparison of gene expression in controls, primary ovarian carcinomas and metastases; (ii) comparison of gene expression in primary ovarian carcinomas and metastases; (iii) survival analysis, i.e., association of gene expression level in intraperitoneal metastases with PFS of patients. $C=$ controls, $\mathrm{PT}=$ primary tumors, $M=$ metastases Red: up-regulation, blue: down-regulation Deregulation of genes in bold remained significant either after the FDR correction or the Cox regression, respectively.

It is important to search for link between various solid tumors and define both common and cancer type-specific markers. In our previous study, we found no difference between $A B C A 7$ gene expression levels in tumors compared to control tissues of colorectal carcinoma (CRC) patients. However; low
$A B C A 7$ expression was associated with poor disease-free survival and higher risk of progression of CRC patients [24]. On the other hand, up-regulation of $A B C A 7$ was previously observed in pancreatic [25] and in breast [26] carcinomas compared to control tissues. Others found $A B C A 7$, in addition to some 
other genes known to be related to cancer progression, up-regulated in chemoresistant ovarian tumors [27]. ABCA7 exports phospholipids and cholesterol from cells to extracellular space [28] and therefore its over-expression in the sequence $\mathrm{C}<\mathrm{pT}<$ $\mathrm{M}$ observed by the present study links the currently discussed role of lipid metabolism in cancer development [29] to its progression. Consequently, the role of $\mathrm{ABCA7}$ in particular tumor types (ovarian or breast carcinoma) needs to be taken into account in future studies.

Ligand-dependent nuclear receptors (e.g., ESR2, NR1H4 and NR1I1) present highly attractive druggable targets [30]. ESR2 is a transcription factor that influences progression of hormone-dependent cancers including ovarian cancer [31]. There is solid evidence that ESR2 expression is decreased in ovarian tumors compared to normal ovaries [32, 33]. Moreover, recently published data showed correlation of ESR2 with EOC risk [34] and OS [35]. Since we observed down-regulation in the order $C>$ pT $>\mathrm{M}$, our data strongly support the role of ESR2 in EOC progression and metastasis.

In addition to deregulation of several genes among controls, primary carcinomas and intraperitoneal metastases, transcript levels of ten genes (ABCA2/8/9/10, ABCB1, ABCC9, ABCG2, $A T P 7 A$, SLC16A14, and SOD3) in EOC metastases were significantly related to PFS in the present study. Out of these, five genes (ABCA9/10, ABCC9, ABCG2 and SLC16A14) confirmed the result of our previous study showing associations of gene expression in primary ovarian carcinomas with PFS of patients [12]. A significant mutual correlation of expression levels of these genes in both primary tumors and metastases, but the lack of such correlation in control tissues observed by the present study suggests existence of specific gene expression profile common to EOC with diagnostic and eventually prognostic potential. Interestingly, $A B C A 10, A B C G 2$ and SLC16A14 were also down-regulated in the order $\mathrm{C}>\mathrm{pT}>\mathrm{M}$, and $A B C C 9$ was up-regulated in metastases compared to primary carcinomas (Figure 3 ).

Several members of ABCA family of membrane transporters, including $A B C A 8$ and $A B C A 9$, were recently associated with outcome of EOC patients; however, $A B C A 10$ was not [13]. ABCA10 is one of the least investigated ABCA family members. It might play a role in lipid metabolism similarly to other ABCA transporters [36], but its function in carcinogenesis of ovarian tumors is unknown. Since we found significant association of high $A B C A 10$ gene expression with long PFS, $A B C A 10$ is worth investigating in a follow-up study in conjunction with other members of ABCA family.
The role of ABCC9 in carcinogenesis is also not well understood. Congruently with our previous study [12], ABCC9 was down-regulated in primary tumors compared to control tissues in the present study. However, the opposite trend, i.e., upregulation in metastases compared to primary EOC tumors was found by the present study. Accordingly, the present study also found opposite trend of association between $A B C C 9$ transcript level and PFS of patients with intraperitoneal metastases compared to the previous study on primary EOC tumors [12]. Thus, $A B C C 9$ relevance for EOC prognosis remains ambiguous; according to our opinion it might be stage-dependent as both sets significantly differed only by stage.

Contrary to some other previously discussed genes, the function of ABCG2 in ovarian cancer cells has been intensively studied. Based mostly on in vitro experiments, several authors have described the relationship between $A B C G 2$ expression and drug resistance [11]. However, Auner et al. failed to observe any connection between $A B C G 2$ expression and resistance in ovarian carcinoma patients [37], which is in agreement with our observations. Similarly, Hedditch et al. observed no relationship either between EOC patient's PFS or OS and ABCG2 expression [13]. Congruently, although the relationship between $A B C G 2$ gene expression and PFS of EOC patients in the present study was significant by the log-rank test, the analysis of hazard risk by the Cox regression test was nonsignificant as it was in the case of $A B C B 1$.

SLC16A14 belongs to the group of monocarboxylate transporters [38]. It is downregulated in ovarian tumors compared to normal tissues [39], and it is related to drug resistance of ovarian cancer cells in vitro [11]. We did not corroborate the role of SLC16A14 in drug resistance of ovarian cancer; however, we found association of high SLC16A14 expression level with longer PFS. The investigation of the role of SLC16A14 in EOC should thus focus not only on drug resistance but also on therapeutic outcomes.

The results of our study did not confirm the previously suggested $[5,7,9]$ role of EGFR, ERBB2, MKI67 or TP53 genes in aggressiveness and progression of EOC.

Taken together, we assume that if validated by follow up studies, particular marker from the present study can be used as a target in neoadjuvant chemotherapy or in the treatment of residual disease after surgery. Moreover, since majority of EOC patients recur, markers (e.g., deregulated genes) specific for metastatic and recurrent disease may then be used for therapy choice in palliative setting. 
The present study had some limitations. Firstly, the study of EOC patients in advanced stages is complicated by the fact that both ovaries are often affected by tumor growth and patients usually present with metastatic spread in the peritoneal cavity. For this reason, we collected control samples of ovaries from an independent group of patients presenting to the hospital for diagnoses other than EOC. Secondly, while there was no difference between age of patients with primary tumors and patients with intraperitoneal metastases, the median age of patients who provided control tissues was considerably lower. This difference is caused by the fact that EOC is usually diagnosed at advanced age, i.e. over 60 years [40]. We cannot exclude, that age-related perturbations in gene expression changes in ovarian tissues of control subjects could influence some of the deregulations observed. Beside this, the modest sample size may be seen as a limitation of the present study.

On the other hand, the present study is unique in the literature as regards number and spectrum of followed genes and especially in the fact that it provides comparison of gene expression level between control ovaries, primary EOCs, and intraperitoneal metastases.

In conclusion, we discovered significant gene expression deregulations among control tissues, primary tumors, and metastases that may potentially contribute to progression of EOC to metastatic disease. We also observed a complex gene expression profile that significantly associated with PFS of patients with intraperitoneal metastases. The above described candidate genes are mainly involved in lipid transport and cell proliferation and may present novel putative molecular markers of prognosis and potential therapeutic targets for ovarian cancer. Potential clinical value of these findings must be verified by larger and independent follow up studies and by the functional characterization of the resulting putative markers.

\section{Supplementary Material}

Supplementary tables. http://www.jcancer.org/v08p3598s1.pdf

\section{Abbreviations}

$\mathrm{ABC}$ transporter: ATP-binding cassette transporter; C: control tissues; CI: confidence interval; CRC: colorectal carcinoma; EMT: epithelialmesenchymal transition; EOC: epithelial ovarian cancer; FDR: false discovery rate; FIGO: International Federation of Gynecology and Obstetrics; HGSC: high grade serous carcinoma; HR: hazard risk; M: intraperitoneal EOC metastases; OS: overall survival;
PFS: progression-free survival; pT: primary EOC tumors; SD: standard deviation; SLC transporter: solute carrier transporter

\section{Acknowledgements}

This study was supported by the Czech Science Foundation (grant no. P303/12/G163), the Ministry of Health of the Czech Republic (project no. 15-25884A), the National Sustainability Program I (NPU I) provided by the Ministry of Education, Youth and Sports of the Czech Republic (grant no. LO1503), and the Charles University Research PROGRES Q 28 (Oncology).

\section{Competing Interests}

The authors have declared that no competing interest exists.

\section{References}

1. Chaffer CL, Weinberg RA. A Perspective on Cancer Cell Metastasis. Science. 2011; 331: 1559-1564.

2. Siegel R, Ma J, Zou Z, et al. Cancer Statistics, 2014. Ca-Cancer J Clin. 2014; 64: 9-29.

3. Nakayama K, Nakayama N, Katagiri H, et al. Mechanisms of Ovarian Cancer Metastasis: Biochemical Pathways. Int J Mol Sci. 2012; 13: 11705-11717.

4. Yeung TL, Leung CS, Yip KP, et al. Cellular and Molecular Processes in Ovarian Cancer Metastasis. A Review in the Theme: Cell and Molecular Processes in Cancer Metastasis. Am J Physiol Cell Physiol. 2015; 309: C444-C456.

5. Colomiere M, Ward AC, Riley C, et al. Cross Talk of Signals between EGFR and IL-6R through JAK2/STAT3 Mediate Epithelial-Mesenchymal Transition in Ovarian Carcinomas. Br J Cancer. 2009; 100: 134-144.

6. Yue P, Zhang X, Paladino D, et al. Hyperactive EGF Receptor, Jaks and Stat3 Signaling Promote Enhanced Colony-Forming Ability, Motility and Migration of Cisplatin-Resistant Ovarian Cancer Cells. Oncogene. 2012; 31: 2309-2322.

7. Serrano-Olvera A, Dueñas-González A, Gallardo-Rincón D, et al. Prognostic, Predictive and Therapeutic Implications of HER2 in Invasive Epithelial Ovarian Cancer. Cancer Treat Rev. 2006; 32: 180-190.

8. Schwartz DR, Kardia SLR, Shedden KA, et al. Gene Expression in Ovarian Cancer Reflects Both Morphology and Biological Behavior, Distinguishing Clear Cell from Other Poor-Prognosis Ovarian Carcinomas. Cancer Res. 2002; 62: 4722-4729.

9. Kamal CK, Simionescu CE, Margaritescu CL, et al. P53 and Ki67 Immunoexpression in Mucinous Malignant Ovarian Tumors. Rom J Morphol Embryol. 2012; 53(Suppl 3): 799-803.

10. Angelopoulou K, Diamandis EP. Detection of the TP53 Tumour Suppressor Gene Product and p53 Auto-Antibodies in the Ascites of Women with Ovarian Cancer. Eur J Cancer. 1997; 33: 115-121.

11. Januchowski R, Zawierucha P, Andrzejewska M, et al. Microarray-Based Detection and Expression Analysis of ABC and SLC Transporters in Drug-resistant Ovarian Cancer Cell Lines. Biomed Pharmacother. 2013; 67: 240-245.

12. Elsnerova K, Mohelnikova-Duchonova B, Cerovska E, et al. Gene Expression of Membrane Transporters: Importance for Prognosis and Progression of Ovarian Carcinoma. Oncol Rep. 2016; 35: 2159-2170.

13. Hedditch EL, Gao B, Russell AJ, et al. ABCA Transporter Gene Expression and Poor Outcome in Epithelial Ovarian Cancer. JNCI. 2014; 106: dju149.

14. Nakayama K, Kanzaki A, Ogawa K, et al. Copper-Transporting P-Type Adenosine Triphosphatase (ATP7B) as a Cisplatin Based Chemoresistance Marker in Ovarian Carcinoma: Comparative Analysis with Expression of MDR1, MRP1, MRP2, LRP and BCRP. Int J Cancer. 2002; 101: 488-495.

15. Samimi G, Safaei R, Katano K, et al. Increased Expression of the Copper Efflux Transporter ATP7A Mediates Resistance to Cisplatin, Carboplatin, and Oxaliplatin in Ovarian Cancer Cells. Clin Cancer Res. 2004; 10: 4661-4669.

16. Moreno-Smith M, Halder JB, Meltzer PS, et al. ATP11B Mediates Platinum Resistance in Ovarian Cancer. J Clin Invest. 2013; 123: 2119-2130.

17. Shen Z, Zhang X, Tang J, et al. The Coupling of Epidermal Growth Factor Receptor Down Regulation by 1alpha,25-Dihydroxyvitamin D3 to the Hormone-Induced Cell Cycle Arrest at the G1-S Checkpoint in Ovarian Cancer Cells. Mol Cell Endocrinol. 2011; 338: 58-67.

18. Swales KE, Korbonits M, Carpenter R, et al. The Farnesoid X Receptor Is Expressed in Breast Cancer and Regulates Apoptosis and Aromatase Expression. Cancer Res. 2006; 66: 10120-10126. 
19. Soucek P, Anzenbacher P, Skoumalova I, et al. Expression of Cytochrome P450 Genes in CD34+ Hematopoietic Stem and Progenitor Cells. Stem Cell. 2005; 23: 1417-1422.

20. Bustin SA, Benes V, Garson JA, et al. The MIQE Guidelines: Minimum Information for Publication of Quantitative Real-Time PCR Experiments. Clin Chem. 2009; 55: 611-622.

21. Benjamini Y, Hochberg Y. Controlling the False Discovery Rate: A Practical and Powerful Approach to Multiple Testing. J R Statist Soc B. 1995; 57: 289-300.

22. [Internet] FDR online calculator. http://www.sdmproject.com/utilities/ ?show $=$ FDR

23. Cassidy J, Bissett D, Spence RAJ, et al. Oxford Handbook of Oncology, third edition. New York, USA: Oxford University Press; 2010.

24. Hlavata I, Mohelnikova-Duchonova B, Vaclavikova R, et al. The Role of ABC Transporters in Progression and Clinical Outcome of Colorectal Cancer. Mutagenesis. 2012; 27: 187-196.

25. Mohelnikova-Duchonova B, Brynychova V, Oliverius M, et al. Differences in Transcript Levels of ABC Transporters between Pancreatic Adenocarcinoma and Nonneoplastic Tissues. Pancreas. 2013; 42: 707-716.

26. Hlaváč V, Brynychová V, Václavíková R, et al. The Expression Profile of ATP-binding Cassette Transporter Genes in Breast Carcinoma. Pharmacogenomics. 2013; 14: 515-529.

27. Bachvarov D, L’Esperance S, Popa I, et al. Gene Expression Patterns of Chemoresistant and Chemosensitive Serous Epithelial Ovarian Tumors with Possible Predictive Value in Response to Initial Chemotherapy. Int J Oncol. 2006; 29: 919-933.

28. Abe-Dohmae S, Ikeda Y, Matsuo M, et al. Human ABCA7 Supports Apolipoprotein-Mediated Release of Cellular Cholesterol and Phospholipid to Generate High Density Lipoprotein. J Biol Chem. 2004; 279: 604-611.

29. Beloribi-Djefaflia S, Vasseur S, Guillaumond F. Lipid Metabolic Reprogramming in Cancer Cells. Oncogenesis. 2016; 5: e189.

30. Lin CY, Gustafsson JA. Targeting Liver X Receptors in Cancer Therapeutics. Nat Rew Cancer. 2015; 15: 216-224.

31. Thomas C, Gustafsson JA. The Different Roles of ER Subtypes in Cancer Biology and Therapy. Nat Rev Cancer. 2011; 11: 597-608.

32. Brandenberger AW, Tee MK, Jaffe RB. Estrogen Receptor Alpha (ER- $\alpha$ ) and Beta (ER- $\beta$ ) mRNAs in Normal Ovary, Ovarian Serous Cystadenocarcinoma and Ovarian Cancer Cell Lines: Down-Regulation of ER- $\beta$ in Neoplastic Tissues. JCEM. 1998; 83: 1025-1028.

33. Suzuki F, Akahira J, Miura I, et al. Loss of Estrogen Receptor $\beta$ Isoform Expression and Its Correlation with Aberrant DNA Methylation of the 5'-Untranslated Region in Human Epithelial Ovarian Carcinoma. Cancer Sci. 2008; 99: 2365-2372.

34. Delfino KR, Rodriguez-Zas SL. Transcription Factor-MicroRNA-Target Gene Networks Associated with Ovarian Cancer Survival and Recurrence. PLOS One. 2013; 8: e58608.

35. Fekete T, Rásó E, Pete I, et al. Meta-Analysis of Gene Expression Profiles Associated with Histological Classification and Survival in 829 Ovarian Cancer Samples. Cancer Genet. 2012; 131: 95-105.

36. Ween MP, Armstrong MA, Oehler MK, et al. The Role of ABC Transporters in Ovarian Cancer Progression and Chemoresistance. Crit Rev Oncol Hematol. 2015; 96: 220-256.

37. Auner V, Sehouli J, Oskay-Oezcelik G, et al. ABC Transporter Gene Expression in Benign and Malignant Ovarian Tissue. Gynecol Oncol. 2010; 117: 198-201.

38. Morris ME, Felmlee MA. Overview of the Proton-Coupled MCT (SLC16A) Family of Transporters: Characterization, Function and Role in the Transport of the Drug of Abuse $\gamma$-Hydroxybutyric Acid. AAPS J. 2008; 10: 311-321.

39. Nikolova DN, Doganov N, Dimitrov R, et al. Genome-Wide Gene Expression Profiles of Ovarian Carcinoma: Identification of Molecular Targets for the Treatment of Ovarian Carcinoma. Mol Med Rep. 2009; 2: 365-384.

40. Jayson GC, Kohn EC, Kitchener HC, et al. Ovarian Cancer. Lancet. 2014; 384: 1376-1388. 